InnOvaciOnes de NegOciOs 17(33): 128-144

(c) 2020 UANL, Impreso en México (ISSN: 2007-1191)

Recepción: 14 Octubre de 2019 Aceptación: 8 Enero de 2020

\title{
Estimación de la función de recaudación tributaria del Instituto Mexicano del Seguro Social sin el apoyo económico gubernamental (Estimate of the tax collection's function of the Mexican Social Security Institute not including of government economic support)
}

\author{
Mauricio Belendez Castañeda \\ UANL Facultad de Contaduría Pública y Adminsitración \\ mauriciobelendez@hotmail.com
}

\begin{abstract}
This paper proposes a statistical model of multiple linear regression (MLR) for decision-making on tax collection, applying it to the Mexican social security system, considering the factors with higher incidence on income perception. The model applied has statistical significance, along with the explanatory variables: number of workers and employers affiliated to the Mexican Social Security Institute (IMSS), contribution percentage (in worker and employer quotas), as well as the existing rate of informality, this at a confidence level of $90 \%$. The fundamental contribution of this article is to propose a statistical model as a financial management tool for the state, in regard to the collection conditions and the institute financing sources, since it was determined from the operational conditions of the social security in Mexico.
\end{abstract}

Key words: Mexican Social Security Institute, Multiple Linear Regression, Social Security Tax Collection.

JEL: E17, H55 y J10

Resumen: El presente trabajo propone un modelo estadístico de regresión lineal múltiple (RLM) para la toma de decisiones sobre la recaudación tributaria con aplicación al sistema de seguridad social mexicano considerando los factores con mayor incidencia en la percepción de ingreso. El modelo aplicado tiene significancia estadística, así como las variables explicativas: número de trabajadores y de patrones afiliados al Instituto Mexicano del Seguro Social (IMSS), porcentaje de aportación (en cuotas del trabajador y empleador), tal como la tasa de informalidad existente, esto en un nivel de confianza del $90 \%$. La contribución fundamental de este artículo es proponer un modelo estadístico como una herramienta de

\section{Estimación de la función de recaudación tributaria}


gestión financiera para el estado, en relación a las condiciones de recaudación y las fuentes de financiamiento del instituto, puesto que fue determinado a partir de las condiciones operativas de la seguridad social en México.

Palabras clave: Instituto Mexicano del Seguro Social, Recaudación Tributaria, Regresión Lineal Múltiple, Seguridad Social.

\section{Introducción}

El método estadístico de Regresión Lineal Múltiple (RLM) trata de ajustar modelos lineales o linealizables entre una variable dependiente y más de una variable independiente, Montero Granados (2016). La estimación de la recaudación tributaria del IMSS depende de factores "independientes", los cuales pueden llegar a conocerse a través de la elaboración de un estudio, al igual que su incidencia en la percepción del ingreso. Lo anterior generaría un modelo con un grado de confiabilidad que pueda ser utilizado para una toma de decisiones financieras con fundamento, sin olvidar la incertidumbre presente en toda toma de decisiones de carácter financiero.

El Instituto Mexicano del Seguro Social (IMSS) es una institución del gobierno federal, autónoma y tripartita (Estado, Patrones y Trabajadores), dedicada a bringar servicios de salud y segurdad social a la población que cuente con afiliación al propio instituto llamados entonces asegurados 0 derechohabientes. La función de RLM se propone de la siguiente forma:

$$
Y=\beta_{0}+\beta_{1} X_{1}+\beta_{2}+\beta_{3}+\beta_{4}+\beta_{5}+\varepsilon
$$

Donde la variable $(Y)$ dependiente es la recaudación y variables independientes (X1, X2, ..., X5) a) número de trabajadores y patrones afiliados al IMSS b) porcentaje de aportación en cuotas del trabajador y empleador, así como la c) tasa de informalidad. En las variables mencionadas no se contempla la aportación gubernamental, ya que se busca proponer nuevas alternativas para sanar tanto la estructura como las finanzas de la seguridad social mexicana con la intervención mínima del apoyo económico por parte del gobierno, encaminados a mejorar las políticas fiscales como lo proponen Jorge Chávez (2000) y Mario Budebo (2000).

Si se analiza el desarrollo de la seguridad social en México, así como el esquema de protección social, se pueden encontrar una serie de dificultades

\section{Belendez, M.}


que se han presentado con el paso del tiempo. Sánchez Vargas (1963), Borgaro (1989) y López (1996), mencionan una serie de inconvenientes, como la falta de uniformidad de las prestaciones, las limitaciones de los fondos de pensiones, el problema de la calidad en la protección social creada dentro del IMSS, la existencia de infraestructura deficiente, así como el factor demográfico y epidemiológico.

Sin embargo, existe un problema crónico de financiamiento, como lo indica Gómez (1966), el déficit económico previsto en la rama de servicios de salud del IMSS es alarmante, debido a que a partir del año 2020 se haría muy probable e incluso necesario el uso de las reservas del instituto según los informes al Ejecutivo Federal y al Congreso de la Unión sobre la Situación Financiera y los Riesgos del IMSS en los años (2017-2018) y (2018-2019), hecho que a corto plazo parece una solución. A largo plazo haría inoperante el servicio de seguridad social en sus cincos seguros: gastos médicos, enfermedades y maternidad, invalidez y vida, riesgos de trabajo y jubilación y cesantía.

En esta investigación el objetivo que persigue es el desarrollo de un modelo de RLM que identifique la relación lineal entre la variable dependiente, la recaudación, con las variables independientes a) número de trabajadores y patrones afiliados al IMSS, b) porcentaje de aportación en cuotas del trabajador y empleador, así como la c) tasa de informalidad, para brindar una alternativa que aumente la recaudación. En este trabajo se busca probar y conocer el grado de incidencia de las variables explicativas seleccionadas en el ingreso recaudado por el instituto, independientemente del aporte gubernamental y así poder brindar una alternativa que aumente la recaudación.

Factores seleccionados para la ecuación de regresión lineal múltiple

El respaldo financiero es de gran relevancia para que el IMSS pueda hacer frente a sus responsabilidades, ya que es un medio para lograr las encomiendas en materia de salud que le corresponden.

Según Frenk et al. (1994), Xu et al. (2003) y Knaul et al (2003), se conocen tres mecanismos básicos para llevar a cabo el financiamiento de los servicios de salud primero de ellos es la recaudación de estado mediante impuestos generales y específicos), el segundo, las cotizaciones a la

\section{Estimación de la función de recaudación tributaria}


seguridad social (recaudadas a través del impuesto sobre la nómina e impuestos de otra índole) y, por último, los pagos privados (pagos directos y pagos de primas de seguros privados).

El Diario Oficial de la Federación (DOF) da a conocer el origen de los ingresos del instituto, los cuales tienen principalmente tres fuentes:

i. De las cuotas y aportaciones tripartitas a la seguridad social que se reciben por parte de los trabajadores, de los patrones y del Gobierno Federal incluidas las multas recargos y actualizaciones.

ii. De los ingresos derivados de las ventas y servicios en tiendas, centros vacacionales, valatorios y la Unidad de Congresos del Centro Médico Siglo XXI.

iii. De los intereses y rendimientos obtenidos de la inversión de las reservas y disponibilidades, así como del uso de los recursos de las reservas y fondos del instituto.

Haciendo uso del banco de datos sobre el desempeño de algunas variables relevantes de la institución del ámbito médico y no médico. Su explotación se realiza bajo el concepto de consulta dinámica (CUBOS de información del IMSS) y para objeto de este estudio, se tomará como variable, y la recaudación al ingreso obrero-patronal (incluye IMSS como patrón y con cifras nominales en millones de pesos). El IMSS es un organismo que tiene carácter fiscal autónomo. Las cuotas obrero-patronales son las aportaciones establecidas en la Ley a cargo del patrón, trabajador y sujetos obligados. Las series que ya no se actualizan no son comparables con la serie a partir de 1997, derivado de la reforma de la Ley del Seguro Social que entró en vigor en ese año.

Un Agente importante para explicar el ingreso del Instituto es el número de empleadores o patrones de los cuales sus registros patronales son determinados conforme a los CUBOS de información del IMSS como una clave interna utilizada en este para identificar a los patrones afiliados. Estas claves no necesariamente corresponden a una empresa, ya que una misma empresa puede tener más de un registro patronal, y tampoco se refiere a un establecimiento, ya que dos o más establecimientos pueden presentar la misma clave patronal. En la cifra de registros patronales se considera a los patrones con al menos un puesto de trabajo afiliado y vigente, y solo incluye

\section{Belendez, M.}


registros no convencionales, ya que los convencionales no están asociados a empresas o establecimientos.

Otro factor que influye en la recaudación es el número de trabajadores, el cual también está proporcionado dentro de los CUBOS de información del IMSS como los asegurados en empleos permanentes y eventuales: La Ley de Seguro Social (LSS) contempla la afiliación en empleos permanentes y eventuales, y en ambos casos, las condiciones de entero y pago de las cuotas al IMSS son las mismas. La única diferencia radica en que los afiliados en empleos clasificados como eventuales requieren de un mayor tiempo previo de aseguramiento para el pago subsidio en caso de enfermedad (LSS, artículos 96 y 97). Un asegurado en empleo permanente es aquél asociado a puestos de trabajo por tiempo indeterminado en los términos de la Ley Federal del Trabajo (artículo 5A, fracción $\mathrm{VI}$ ); asegurado en empleo eventual es aquél asociado a un puesto de trabajo para obra determinada o por tiempo determinado en los términos de la Ley Federal del Trabajo (LSS, artículo 5A, fracción VII).

Además de los mencionados, se agrega la tasa de informalidad laboral tomada del DOF como la proporción de la población ocupada que comprende a la suma, sin duplicar, de los ocupados que son laboralmente vulnerables por la naturaleza de la unidad económica para la que trabajan con aquellos cuyo vínculo o dependencia laboral no es reconocido por su fuente de trabajo.

Por último, uno de los factores más interesantes son el porcentaje de aportación en cuotas por parte del patrón y el trabajador, tal como se establece en el DOF, es la cantidad de dinero que se paga al IMSS, por los trabajadores, patrones y la cuota social que subsidia el gobierno mexicano. La forma de calcular dicha cuota, es en base al salario base cotización que se informó al IMSS cuando causó alta el trabajador, asimismo, se consideran los porcentajes que fija la LSS.

\section{Metodología}

Para aplicar el análisis de RLM, se recolectaron los datos del periodo de tiempo 1997-2017 de México (Tablas 1 y 2), y con el fin de aumentar la aceptación de los supuestos que deben de cumplir los datos, se les da el siguiente tratamiento:

\section{Estimación de la función de recaudación tributaria}


- $\quad$ "Y"="REC" (variable de respuesta): Ingreso obrero-patronal anual acumulado (CUBOS de información del IMSS), cifra en millones de pesos.

- $\quad$ "X1"="Trab/Pat" (variable regresora): División entre el número de asegurados asociados a un empleo eventual y/o permanente (CUBOS de información de IMSS) y el número de patrones asociados a asegurados (CUBOS de información del IMSS).

- $\quad$ "X2"="Prom T1, T2" (variable regresora) ("T1" trabajador tipo uno: hasta un salario mínimo y "T2" trabajador tipo dos: hasta tres salarios mínimos): promedio de porcentaje de aportación del trabajador tipo uno y dos (DOF).

- "X3"="Prom T3, T4 y T5" (variable regresora) ("T3" trabajador tipo tres: hasta cinco salarios mínimos, "T4" trabajador tipo cuatro: hasta diez salarios mínimos y "T5" trabajador del tipo cinco: hasta veinticinco salarios mínimos, tope salarial por ley): promedio de porcentaje de aportación del trabajador tipo tres, cuatro y cinco (DOF).

- "X4"="Prom P'S" (variable regresora) ("P1" patrón tipo uno: hasta un salario mínimo, "P2" patrón tipo dos: hasta tres salarios mínimos, "P3" patrón tipo tres: hasta cinco salarios mínimos, "P4" patrón tipo cuatro: hasta diez salarios mínimos y "P5" patrón tipo cinco hasta veinticinco salarios mínimos, tope salarial por ley): promedio de las cinco categorías de porcentaje de aportación del patrón (DOF).

- "X5"="Inform" tasa de informalidad correspondiente al periodo (DOF). Se aplica LOG 10 a cada una de las variables, transformando toda la serie de datos y así mejorar las premisas de linealidad.

Tabla 1. Recaudación (cifra en millones de pesos), tasa de informalidad, número de trabajadores y empleadores

\begin{tabular}{lllll}
\hline Año & REC & Patrón & Trabajador & Inform. \\
\hline 1997 & 52,378 & 693,985 & $10,536,717$ & 0.6287 \\
1998 & 52,937 & 715,453 & $11,243,230$ & 0.6054 \\
1999 & 65,841 & 744,562 & $11,905,112$ & 0.6086 \\
2000 & 79,498 & 783,085 & $12,437,760$ & 0.5883 \\
2001 & 89,093 & 806,464 & $12,170,945$ & 0.5857 \\
2002 & 95,423 & 811,307 & $12,232,301$ & 0.5945 \\
2003 & 108,099 & 805,964 & $12,257,581$ & 0.6009 \\
2004 & 115,541 & 802,012 & $12,632,877$ & 0.5971 \\
2005 & 120,730 & 803,848 & $13,061,565$ & 0.5945
\end{tabular}

Belendez, M. 


\begin{tabular}{lllll}
2006 & 131,861 & 816,319 & $13,678,492$ & 0.5837 \\
2007 & 142,469 & 827,833 & $14,207,706$ & 0.5786 \\
2008 & 150,735 & 829,226 & $14,178,117$ & 0.5836 \\
2009 & 151,932 & 822,374 & $14,006,404$ & 0.597 \\
2010 & 162,817 & 826,959 & $14,738,783$ & 0.594 \\
2011 & 180,435 & 827,498 & $15,350,335$ & 0.5948 \\
2012 & 197,194 & 836,215 & $16,062,043$ & 0.5944 \\
2013 & 211,801 & 842,848 & $16,525,061$ & 0.586 \\
2014 & 228,885 & 854,909 & $17,239,587$ & 0.5774 \\
2015 & 248,868 & 885,846 & $17,884,033$ & 0.5785 \\
2016 & 268,836 & 917,680 & $18,616,624$ & 0.5729 \\
2017 & 291,705 & 949,411 & $19,418,455$ & 0.5729 \\
\hline
\end{tabular}

Fuente: Adaptación propia. Número de trabajadores y patrones Cubos de información del IMSS 1997-2017. Recaudación y tasa de informalidad del DOF.

Cumpliendo con los lineamientos de cada año para el periodo 19972017, se utiliza la calculadora "Cálculos cuotas IMSS Obrero-Patronales (2018)" para calcular el porcentaje de aportación en cuotas para el empleador y trabajador (Tabla 2):

Para el cálculo de porcentaje para cada una de las clasificaciones, se usan los siguientes datos:

A. Salario mínimo área geográfica (Integrado)

B. Salario mínimo mensual (Integrado)

C. Para el patrón:

- Enfermedad y maternidad

- Invalidez y vida

- Cesantía en edad avanzada y vejez

D. Para el trabajador:

- Enfermedad y maternidad

- Riesgo de trabajo

- Invalidez y vida

- Guarderías y prestaciones sociales

- Retiro

- Cesantía en edad avanzada y vejez

\section{Estimación de la función de recaudación tributaria}


E. Condicionado por:

- 30 días a cotizar

- Vacaciones

- Aguinaldo

- Prima vacacional

F. Se omite el cálculo de Infonavit

Cálculo acorde a la Ley Federal del Trabajo (LFT), a la LSS y a la Constitución Política de los Estados Unidos Mexicanos.

Se seleccionaron 5 tipos o categorías para trabajador "Tn" y patrón "Pn" (Tabla2):

- $\quad$ "T1" y "P1" Trabajador y Patrón del tipo uno: hasta un salario mínimo.

- "T2" y "P2" Trabajador y Patrón del tipo dos: hasta tres salarios mínimos.

- $\quad$ "T3" y "P3" Trabajador y Patrón del tipo tres: hasta cinco salarios mínimos.

- $\quad$ "T4" y "P4" Trabajador y Patrón del tipo cuatro: hasta diez salarios mínimos.

"T5" y "P5" Trabajador y Patrón del tipo cinco: hasta veinticinco salarios mínimos, tope salarial por ley.

Tabla 2. Porcentaje de aportación en cuotas para el patrón y el trabajador

\begin{tabular}{cllllllllll}
\hline Año & T1 & T2 & T3 & T4 & T5 & P1 & P2 & P3 & P4 & P5 \\
\hline 1997 & 0.022 & 0.022 & 0.0397 & 0.0397 & 0.0397 & 0.2587 & 0.1766 & 0.2134 & 0.2011 & 0.1937 \\
1998 & 0.0221 & 0.0221 & 0.0391 & 0.0391 & 0.0391 & 0.2626 & 0.1783 & 0.2126 & 0.2 & 0.1924 \\
1999 & 0.0221 & 0.0221 & 0.0384 & 0.0384 & 0.0384 & 0.2962 & 0.21 & 0.2417 & 0.2288 & 0.221 \\
2000 & 0.0221 & 0.0221 & 0.037 & 0.037 & 0.037 & 0.3021 & 0.212 & 0.2386 & 0.2251 & 0.217 \\
2001 & 0.0224 & 0.0224 & 0.0361 & 0.0361 & 0.0361 & 0.3123 & 0.217 & 0.2388 & 0.2245 & 0.2159 \\
2002 & 0.0226 & 0.0226 & 0.035 & 0.035 & 0.035 & 0.3217 & 0.2213 & 0.2381 & 0.2231 & 0.214 \\
2003 & 0.0228 & 0.0228 & 0.0338 & 0.0338 & 0.0338 & 0.3301 & 0.225 & 0.2366 & 0.2209 & 0.2114 \\
2004 & 0.0229 & 0.0229 & 0.0325 & 0.0325 & 0.0325 & 0.3379 & 0.2282 & 0.2346 & 0.2181 & 0.2082 \\
2005 & 0.023 & 0.023 & 0.0312 & 0.0312 & 0.0312 & 0.3454 & 0.2312 & 0.2323 & 0.2151 & 0.2049 \\
2006 & 0.023 & 0.023 & 0.0297 & 0.0297 & 0.0297 & 0.3514 & 0.2332 & 0.2289 & 0.2112 & 0.2006 \\
2007 & 0.023 & 0.023 & 0.0282 & 0.0282 & 0.0282 & 0.3575 & 0.2353 & 0.2256 & 0.2072 & 0.1962 \\
2008 & 0.023 & 0.023 & 0.0268 & 0.0268 & 0.0268 & 0.3635 & 0.2373 & 0.2222 & 0.2033 & 0.1919 \\
2009 & 0.0231 & 0.0231 & 0.0268 & 0.0268 & 0.0268 & 0.3647 & 0.2381 & 0.223 & 0.204 & 0.1926
\end{tabular}

Belendez, M. 


\begin{tabular}{lllllllllll}
2010 & 0.0231 & 0.0231 & 0.0268 & 0.0268 & 0.0268 & 0.3647 & 0.238 & 0.223 & 0.204 & 0.1926 \\
2011 & 0.0231 & 0.0231 & 0.0268 & 0.0268 & 0.0268 & 0.3647 & 0.238 & 0.2229 & 0.204 & 0.1926 \\
2012 & 0.023 & 0.023 & 0.0267 & 0.0267 & 0.0267 & 0.3632 & 0.2371 & 0.222 & 0.2031 & 0.1918 \\
2013 & 0.0242 & 0.0242 & 0.0281 & 0.0281 & 0.0281 & 0.3822 & 0.2495 & 0.2337 & 0.2138 & 0.2018 \\
2014 & 0.0238 & 0.0238 & 0.0276 & 0.0276 & 0.0276 & 0.3749 & 0.2447 & 0.2292 & 0.2097 & 0.198 \\
2015 & 0.0233 & 0.0233 & 0.027 & 0.027 & 0.027 & 0.3674 & 0.2398 & 0.2246 & 0.2055 & 0.194 \\
2016 & 0.0238 & 0.0238 & 0.0276 & 0.0276 & 0.0276 & 0.3747 & 0.2446 & 0.2291 & 0.2096 & 0.1979 \\
2017 & 0.0238 & 0.0238 & 0.0276 & 0.0276 & 0.0276 & 0.3747 & 0.2446 & 0.2291 & 0.2096 & 0.1979 \\
\hline
\end{tabular}

Fuente: Elaboración propia. Utilizando la calculadora "Cálculos cuotas IMSS Obrero-Patronales (2018)" se calculan los porcentajes de aportación en cuotas para el empleador y trabajador. Cálculo acorde a la Ley Federal del Trabajo (LFT), a la Ley del Seguro Social (LSS) y a la Constitución Pólitica de los Estados Unidos Mexicanos. Omisión del cálculo de Infonavit.

\section{Pruebas del modelo de RLM}

El modelo de RLM es una técnica estadística que servirá como herramienta para calcular la similitud entre la variable dependiente, la recaudación, con las variables independientes, esta relación será representada en forma de función matemática. En la construcción de una función eficiente es necesario el análisis de residuales, por lo tanto, se deben examinar los residuos, que, en el contexto de la regresión lineal, se les llamará residuo o error a las diferencias entre los valores observados de la variable y los valores que se predicen a partir de la recta de regresión de este estudio (Gráfica 1).

Como herramienta para determinar si el modelo de RLM es apropiado para la selección de datos, dado que se cuenta con distintas variables, se deberán de correr varias pruebas de residuos; es usual graficar los residuos contra los valores predichos de la variable dependiente y para este estudio serán asignados como recaudación estimada.

El estudio de residuales consiste en utilizar los datos de la muestra y verificar mediante pruebas de hipótesis si se puede o no rechazar la hipótesis nula, para este procedimiento es necesario asignar un error alfa (a), valor comprendido entre 0 y 1 que mide cuánto se equivoca el modelo al aceptar como verdadera la hipótesis alternativa de un test. Cuando más pequeño sea el error alfa, más precisos serán los resultados. En la función de predicción de este estudio, se fijará el error en 0.10 .

\section{Estimación de la función de recaudación tributaria}


Análisis de normalidad de los residuales

En la RLM, llamamos residuos a las diferencias entre los valores de la variable dependiente observados y los valores que predecimos a partir de nuestra recta de regresión (Gráfica 1).

Gráfica 1. Recaudación real vs recaudación estimada 1997-2017

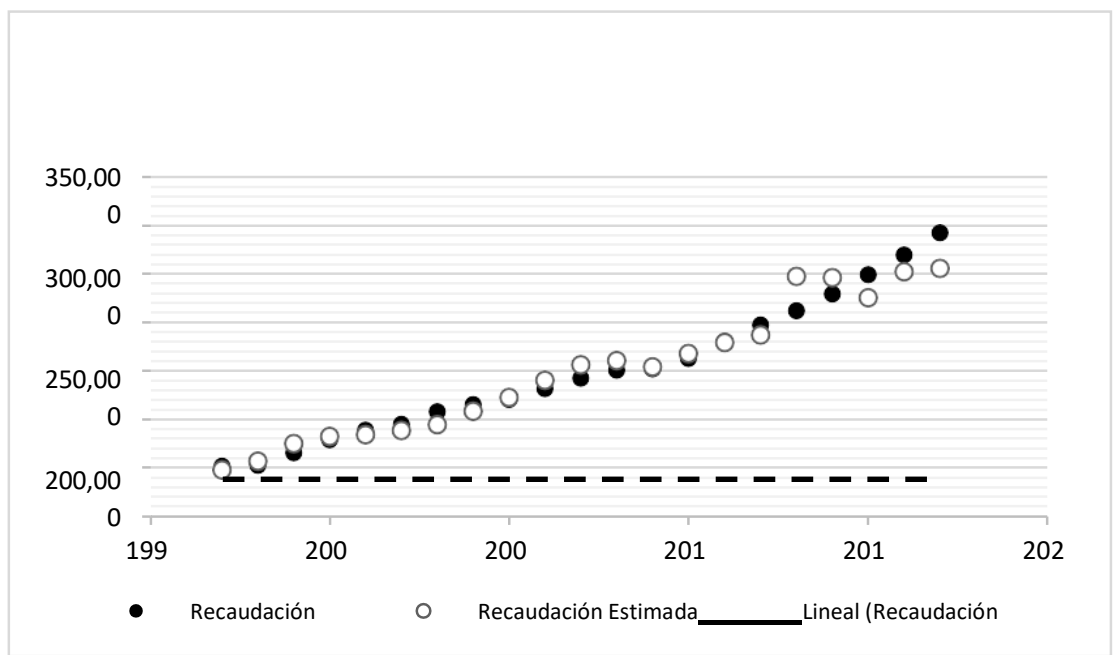

Fuente: Elaboración propia apoyado con el software Minitab, datos de CUBOS de Información del IMSS 1977-2017. Para la recaudación real y para la recaudación estimada se utiliza el modelo de regresión

El histograma de residuos (Gráfica 2), muestra la distribución de los residuos para todas las observaciones. El histograma de los residuos es utilizando para todas las observaciones. El histograma de los residuos es utilizado para determinar si los datos son asimétricos o incluyen valores atípicos. Los patrones pueden indicar si el modelo cumple con las premisas del modelo.

Belendez, M. 
Gráfica 2. Histograma de residuales

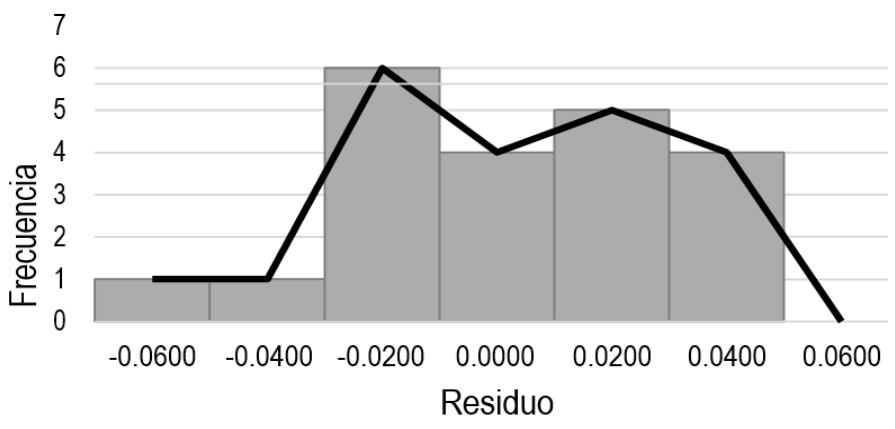

Fuente: Elaboración propia

Una prueba de hipótesis examina dos hipótesis opuestas sobre una población: la hipótesis nula $(\mathrm{HO})$ y la hipótesis alternativa $(\mathrm{Ha})$. La hipótesis nula es el enunciado que se probará, que para efecto de nuestro estudio seria probar la normalidad de la distribución de los residuales (Tabla 3).

Tabla 3. Prueba Normalidad de Residuales

\begin{tabular}{lll}
\hline $\mathrm{p}$-valor & \multicolumn{1}{c}{$\boldsymbol{H}_{\mathbf{0}}$} & \multicolumn{1}{c}{$\boldsymbol{H}_{\boldsymbol{a}}$} \\
\hline 0.433 & Los errores se distribuyen normalmente & Los errores no se distribuyen normalmente \\
$0.433<\mathrm{a}=0.1$ & No cumple & No se rechaza $H_{0}$ \\
\hline Fuente: &
\end{tabular}

Fuente: Elaboración propia apoyado con el software Minitab.

\section{Análisis de varianza}

El análisis de varianza consiste en hacer pruebas de hipótesis individuales para cada variable regresora y así conocer el grado de influencia de cada una de ellas en la recaudación (Tabla 4 y 5).

Tabla 4. Análisis de Varianza

\begin{tabular}{lccccc}
\hline Fuente & gl & SC ajust. & MC ajust. & Valor F & $\begin{array}{c}\text { Valor } \\
\mathbf{p}\end{array}$ \\
\hline Regresión & 5 & 0.946703 & 0.189341 & 105.61 & 0 \\
LOG (10) & 1 & 0.029625 & 0.029625 & 16.52 & 0.001 \\
Trab/Pat: & & & & & \\
LOG (10) & 1 & 0.005626 & 0.005626 & 3.14 & 0.097 \\
Prom T1, T2: & & & & 12.21 & 0.003 \\
LOG (10) & 1 & 0.021896 & 0.021896 & & \\
Prom T3, T4 y & & & & &
\end{tabular}

Estimación de la función de recaudación tributaria 


$\begin{array}{lccccc}\text { T5: } & & & & \\ \text { LOG (10) } & 1 & 0.008708 & 0.008708 & 4.86 & 0.044 \\ \text { Promedio } & & & & & \\ \text { P'S: } & & & & \\ \quad \text { LOG (10) } & 1 & 0.002849 & 0.002849 & 1.59 & 0.227 \\ \quad \text { Inform.: } & & & & \\ \quad \text { Error: } & 15 & 0.026893 & & & \\ \quad \text { Total: } & 20 & 0.973596 & & \end{array}$

Tabla 5. Prueba de varianza para las Betas $\beta$

\begin{tabular}{|c|c|c|c|c|}
\hline p-valor & $\begin{array}{l}\text { Prueba de } \\
\text { hipótesis }\end{array}$ & Variable & $\mathrm{H}_{0}$ & $\boldsymbol{H}_{\boldsymbol{a}}$ \\
\hline 0 & $0<a=0.1$ & & $\begin{array}{l}\beta_{0}=\beta_{1}=\beta_{2}=\beta_{3}=\beta_{4}= \\
\beta_{5}=0\end{array}$ & \\
\hline 0.001 & $0.001<.1$ & $X_{1}$ Trab/Pat & & \\
\hline 0.097 & $0.097<.1$ & $\begin{array}{l}X_{2} \text { Prom. T1 } \\
\text { y T2 }\end{array}$ & & \\
\hline 0.003 & $0.003<.1$ & $\begin{array}{l}X_{3} \text { Prom. T3, } \\
\text { T4, y T5 }\end{array}$ & $X_{i} \beta_{i}=0$ (No influye en & $\begin{array}{c}X_{i} \beta_{i} \text { diferente } \\
\text { de } 0\end{array}$ \\
\hline 0.044 & $0.044<.1$ & $\begin{array}{l}X_{4} \text { Prom. P1, } \\
\text { P2, P3, P4 y } \\
\text { P5 }\end{array}$ & & \\
\hline 0.227 & $0.227<.1$ & $X_{5}$ Inform. & & \\
\hline
\end{tabular}

Fuente: Elaboración propia.

Independencia entre variables

Análisis de multicolinealidad, Factor de Inflación entre Variables

(FIV), si el FIV < 10 no existe correlación entre las variables, cumpliéndose el supuesto de variables independientes (Tabla 6).

Tabla 6. Independencia entre variables

\begin{tabular}{lrrrrrr}
\hline Termino & Coeficiente & $\begin{array}{l}\text { Estimación } \\
\text { de error del } \\
\text { coeficiente }\end{array}$ & Valor T & Valor p & FIV & \\
\hline Constante & 13.86 & 3.5 & 3.96 & 0.001 & \\
LOG (10) & 1.661 & 0.409 & 4.06 & 0.001 & 4.23 \\
Trab/Pat: & & & & &
\end{tabular}

Belendez, M. 


$\begin{array}{lccccc}\text { LOG (10) } & 3.51 & 1.98 & 1.77 & 0.097 & 5.85 \\ \text { Prom T1, } & & & & & \\ \text { T2: } & & & & \\ \text { LOG (10) } & -1.043 & 0.299 & -3.49 & 0.003 & 4 \\ \text { Prom T3, T4 } & & & & & \\ \text { y T5: } & 1.744 & 0.791 & 2.2 & 0.044 & 3.45 \\ \text { LOG (10) } & & & & & \\ \text { Promedio } & & 1.64 & -1.26 & 0.227 & 2.77 \\ \text { P'S: } & -2.07 & & & \\ \text { LOG (10) } \\ \text { Inform.: }\end{array}$

\section{Resultados}

1) Los residuales se distribuyen de forma normal.

2) Las variables $X_{1}$ Trab./ Pat., $X_{2}$ Prom. T1 y T2, $X_{3}$ Prom. T3, T4 y T5, $X_{4}$ Prom. De P'S influyen en el modelo. La variable $X_{5}$ Inform. no influye en el modelo, pero se deja dentro de la ecuación ya que mejora el ajuste a la línea de regresión.

3) Basados en el análisis de independencia entre variables regresoras se puede concluir que no hay correlación entre variables regresoras

4) El modelo estimado se presenta a continuación:

$L O G(10) R E C=13.86+1.661 L O G(10)$ Trab $/$ Pat + 3.51LOG(10)PromT1, T2 - 1.043LOG(10)PromT3, T4yT5 + 1.744LOG(10) PromP'S - 2.07 LOG(10)Inform.

5) Resumen del modelo

Tabla 7. Resumen del modelo

\begin{tabular}{llll}
\hline $\mathbf{S}$ & R-cuad. & $\begin{array}{l}\text { R-cuad. } \\
\text { (ajustado) }\end{array}$ & $\begin{array}{l}\text { R-CUAD. } \\
\text { (pred) }\end{array}$ \\
\hline 0.0423425 & $97.24 \%$ & $96.32 \%$ & $91.97 \%$ \\
\hline
\end{tabular}

\section{Estimación de la función de recaudación tributaria}


a) Desviación estándar "S": .0423. El modelo posee una desviación estándar relativamente aceptable, teniendo en cuenta que mientras la desviación estándar sea mayor, la dispersión de los datos también aumentará y una dispersión alta aleja a los puntos de la línea de regresión, lo que afectaría directamente la ecuación de predicción y disminuiría su confiabilidad.

b) Confiabilidad del ajuste del modelo "R-cuad (ajuste)": $98.61 \%$

c) Confiabilidad de la predicción del modelo "R-cuad (predicción): 91.97\%

6) En el caso de la variable, número de trabajdores, dividido entre el número de empleadores, se observa que existe una relación positiva con la variable dependiente y que el impacto en dicha variable es de un 1.661, lo cual demuestra dos cosas. La primeara es que la recaudación le beneficia numéricamente una mayor cantidad de empleados con respecto al número de empleadores existentes, la segunda es que, por cada patrón afiliado al Instituto, hay aproximadamente 1.66 trabajadores incorporados a este.

7) Para la variable, promedio de porcentaje de aportación del trabajador tipo uno y dos, donde interactúan las clasificaciones "T1" y "T2", hasta uno y tres salarios mínimos respectivamente, se ve que el signo que reporta la regresión es positivo y cuyo parámetro es 3.51, lo que demuestra que, al ser descontado el porcentaje de aportación a los trabajadores dentro de esta designación, influye positivamente a la recaudación en 3.51.

8) Para la variable, promedio de porcentaje de aportación del trabajador tipo tres, cuatro y cinco, donde interactúan las clasificaciones "T3", "T4" y "T5" hasta cinco, diez y veinticinco salarios mínimos respectivamente, se puede observar que tiene un signo negativo, lo cual demuestra que una distribución de los porcentajes de aportación que aumente el promedio haría que disminuya el ingreso percibido de estas clasificaciones.

9) Para el caso de la variable promedio de las cinco categorías de porcentaje de aportación del patrón "P1", "P2", "P3", "P4" y "P5" (patrón del tipo uno, dos, tres, cuatro y cinco), donde cada uno representa hasta uno, tres, cinco, diez, y veinticinco salarios mínimos respectivamente, se pudo llegar a la

\section{Belendez, M.}


conclusión de que la relación entre esta variable y la recaudación es positiva y el impacto que tiene es de 1.74, esto es que el conjunto de las aportaciones patronales influye un 1.74 en la percepción de ingreso.

10) Para el caso de la variable tasa de informalidad, se puede mencionar que no cumple la prueba significancia, pero se deja dentro del modelo ya que favorece al cumplimiento de los supuestos de normalidad de los residuales mencionados con anterioridad.

\section{Discusión}

En este trabajo se comprueba la existencia de la relación estadística entre la variable dependiente, la recaudación tributaria del IMSS, con las variables independientes a) número de trabajadores y patrones afiliados al IMSS y b) porcentaje de aportación en cuotas del trabajador y empleador, siendo significantes en un $90 \%$. Sin embargo, el factor c) tasa de informalidad, no cumple con el nivel de confiabilidad, pero se deja dentro del modelo y a que favorece al ajuste de la línea de regresión, esto quiere decir que beneficia al cumplimiento de las pruebas de normalidad de los residuales.

Una segunda conclusión es que la variable, número de trabajadores entre número de patrones afiliados al instituto, puede interpretarse como una relación entre estos dos agentes, donde ambos guardan una relación positiva con el ingreso, además de fundamentarse numéricamente, que una mayor cantidad de trabajadores dentro de la formalidad, comparados con el número de empleadores, beneficia a la variable de respuesta.

Las variables que contemplan el porcentaje de aportación por parte de los trabajadores, llevan a la conclusión de que las clasificaciones "T1" y "T2" (hasta 1 y 3 salarios mínimos respectivamente), son las de mayor peso en el modelo, influyendo de manera positiva, lo cual indica que estos contribuyentes son los que más favorecen y aportan a la recaudación del IMSS. Las cuotas restantes de los trabajadores poseen una relación negativa, lo cual expone que una mala distribución de las cuotas por parte de los trabajdores, perjudica la contribución proveniente de los factores "T3", "T4" $Y$ "T5" (hasta 5, 10 y 25 salarios mínimos respectivamente).

El análisis de las cuotas patronales arroja como resultado una relación positiva de estas hacia la recaudación, lo cual lleva a determinar que la

\section{Estimación de la función de recaudación tributaria}


segunda variable, participación por parte de los patrones, es de mayor importancia.

Por otro lado, es relevante la inclusión de la tasa informalidad, dado que es un factor con el cual se podría abundar sobre la relación entre el trabajador y empleador dentro de la formalidad.

Otra conclusión es que al obtener la ecuación de regresión que permita pronosticar valores recaudatorios, también se podría llegar a conocer el comportamiento que deberían de tener los factores independientes, bajo cierto grado de certeza, lo cual consitutye una herramienta poderosa para próximas investigaciones pues se brinda la posibilidad de hacer ajustes en los procesos, en la toma de decisiones o en el establecimiento de políticas.

A pesar de lo importante que resulta ser para cualquier profesional el conocimiento y uso del análisis de RLM, es una herramienta muy poco aprovechada, como lo demuestran gran número de trabajos de grado a nivel posgrado y trabajos de investigación, en los cuales el desarrollo estadístico solo se limita a la parte descriptiva y no a la inferencial.

\section{Referencias}

Centro de Estudios de Finanzas Públicas CEFP (2013). Indicadores sobre seguridad social en México. Disponible en: www.cefp.gob.mx

Cetrángolo, O. y Gómez, J. (2006). Tributación en América Latina. En busca de una nueva agenda de refor,as. Santiago de Chile: Comisión Económica para América Latina y el Caribe (CEPAL).

Consejo Nacional de Población, CONAPO (2018). Proyecciones de población de México y de las entidades federativas 2016-2050. México: Secretaría General del CONAPO.

Departamento de especialistas en estadística de Minitab $(2015,2017)$. Informe técnico sobre el asistente de Minitab. Regresión múltiple.

Díaz, E. (2017). Impuesto al trabajo y distribución del ingreso: ¿Contribuye el impuesto laboral a una mejor distribución del ingreso en los asalariados? Economía UNAM, 4(12).

Guillermo, P., Beatriz, S. y Vargas, I. (2007). Recaudación potencial, eficiencia recaudatoria y transferencias federales: Un análisis para las entidades federativas en México utilizando el modelo de frontera estocástica. EconoQuantum, 14 (1). 35-71.

Instituto de investigaciones legislativas del senado de la república IILSEN (2003). Modelos de recaudación fiscal. Disponible en: www.blbliodigitalibd. Senado.gob.mx

Macías, E. (1993) El sistema de pensiones en México dentro del contexto internacional. México: Instituto de proposiciones estratégicas.

\section{Belendez, M.}


Montero, R. (2016). Modelos de regresión lineal múltiple. España: Documentos de trabajo en Economía Aplicada. Universidad de Granada.

Russel, B. (1931). The Scientific Outlook. Reino Unido, Londres: George Allen \& Union.

Sánchez A. (2012). La seguridad y la protección social en México. Su necesaria reorganización. Estado de México: Instituto de Investigación Jurídica de la UNAM. 